\title{
CARACTERÍSTICAS DE MASTIGAÇÃO E DEGLUTIÇÃO NA DOENÇA DE ALZHEIMER
}

\section{Characteristics of mastication and swallowing in Alzheimer's disease}

\author{
Thaíza Estrela Tavares ${ }^{(1)}$, Cecília Maria Resende Gonçalves de Carvalho ${ }^{(2)}$
}

\begin{abstract}
RESUMO
Objetivos: comparar as características de mastigação e deglutição em idosos com e sem Doença de Alzheimer (DA). Método: estudo comparativo desenvolvido com 86 idosos, na faixa etária entre 60 e 93 anos, de ambos os sexos, distribuídos nos grupos controle (sem DA, $n=43$ ) e experimental (com $\mathrm{DA}, \mathrm{n}=43$ ). A função cognitiva e o estadiamento da DA foram avaliados por meio da aplicação da CDR (Clinical Dementia Rating). Para a caracterização da mastigação e deglutição foi aplicado o protocolo de avaliação miofuncional orofacial. Resultados: as características de mastigação e deglutição sofreram variações importantes quando comparados os dois grupos. Houve diferença significante para movimentos mandibulares, reflexo de deglutição, deglutições múltiplas, presença de resíduos e dificuldade para ingerir comprimidos. Conclusões: as características de mastigação e deglutição, de maneira geral, sofrem maior comprometimento nos idosos com DA, quando comparados a idosos saudáveis.
\end{abstract}

DESCRITORES: Idosos; Doença de Alzheimer; Mastigação; Deglutição

\section{INTRODUÇÃO}

O envelhecimento da população mundial é incontestável. No Brasil, o crescimento da população geriátrica constitui um grande desafio para a saúde pública. Enquanto a população brasileira crescerá 3,22 vezes até o ano 2025 , o segmento acima de 65 anos aumentará 8,9 vezes, e o acima de 80 anos, 15,6 vezes. Projeções indicam que em 2025, ocupará o 6 lugar entre os países com mais idosos no mundo ${ }^{1}$. A transição demográfica vem sendo acompanhada por uma transição epidemiológica, que tem como principal característica a

(1) Fonoaudióloga da Fundação Municipal de Saúde; Professora da Faculdade de Saúde, Ciências Humanas e Tecnológicas do Piauí - NOVAFAPI, Teresina, Piauí, Brasil; Especialização em Motricidade Oral pela Universidade Federal de Pernambuco-UFPE; Mestrado em Ciências e Saúde pela Universidade Federal do Piauí-UFPI.

(2) Nutricionista; Professora Associada I da Universidade Federal do Piauí; Coordenadora do Núcleo de Pesquisa e Extensão Universitária para a Terceira Idade da Universidade Federal do Piauí - UFPI, Teresina, Piauí, Brasil; Doutorado em Ciência da Nutrição pela Universidade Estadual de Campinas.

Conflito de interesses: inexistente crescente incidência de doenças crônico-degenerativas, dentre elas, a Doença de Alzheimer (DA)². Assim, surge a preocupação da comunidade científica com a qualidade de vida da população idosa, criando novas demandas por intervenção especializada, como é o caso da Fonoaudiologia.

A Doença de Alzheimer é uma enfermidade neurodegenerativa progressiva que causa perda da memória e altera funções intelectuais superiores, levando, no curso de sua evolução, a uma situação de incapacidade e total dependência. Em virtude de sua incidência e natureza devastadoras, caracteriza um importante problema de saúde pública em todo o mundo ${ }^{3}$. Por ser uma doença grave e ainda sem cura, gera múltiplas demandas e altos custos financeiros, o que representa um novo desafio para o poder público, instituições, profissionais de saúde, aliás, para todos. A DA caracteriza-se por degeneração que acomete inicialmente a formação hipocampal, o centro de memória de curto prazo. Posteriormente, atinge áreas corticais associativas, havendo relativa preservação dos córtices primários. Compromete a memória, afeta a orientação, atenção, linguagem, capacidade para resolver problemas e habilidades para desempenhar as atividades da vida diária ${ }^{4}$. 
A evolução clínica da DA está dividida em três estágios: inicial/leve, moderado e tardio/severo. As alterações fonoaudiológicas estão inicialmente associadas a dificuldades na comunicação, evoluindo progressivamente para níveis mais elevados de dependência de uma forma geral, incluindo as atividades de vida diária, especialmente a alimentação, com dificuldade marcante na mastigação e deglutição. O doente torna-se totalmente dependente, especialmente para atividades de vida diária, alimentação e higiene pessoal ${ }^{5}$, o que deteriora completamente sua qualidade de vida. Apesar da caracterização específica de cada estágio, a evolução clínica da doença, bem como as alterações e dificuldades decorrentes, são únicas para cada indivíduo 6 .

O idoso portador da DA tem seu estado nutricional afetado de forma drástica, possivelmente por uma provável incapacidade de aceitação, mastigação, deglutição e assimilação dos alimentos. A perda de apetite, o desinteresse pela alimentação e a falta de consciência da importância da nutrição, aumentam os riscos de desidratação e desnutrição, influenciando no quadro de saúde geral ${ }^{7}$. As doenças crônico-degenerativas geralmente afetam as necessidades orgânicas de proteínas e de calorias, podendo estar associadas à inapetência, causada pela própria doença, por determinados medicamentos e por dificuldades de alimentação. Tais dificuldades vão desde falta de auxílio para oferecer as refeições, ausência de dentes, consistência alimentar de difícil deglutição ou não atrativa ao paladar, entre outras. Desta forma, avaliações odontológicas, fonoaudiológicas e nutricionais periódicas são fundamentais para prevenir a desnutrição. A evolução da DA aumenta a perda de peso, atuando como fator determinante de mortalidade para esses casos ${ }^{8}$.

Muitas das alterações observadas no idoso interferem de forma negativa na sua alimentação, e, conseqüentemente, em sua qualidade de vida. As alterações mais frequentemente descritas na literatura são: desgaste ou perda dos dentes, diminuição da força muscular, lentidão mastigatória, diminuição da produção de saliva, alteração na mucosa e epitélio bucal e lingual, diminuição da percepção gustativa e olfativa, diminuição da capacidade para deglutir, entre outras ${ }^{9}$. Quando há uma doença crônica em progresso, o quadro tende a agravar-se ainda mais.

As modificações estruturais e funcionais alteram o processo de mastigação e deglutição, modificando, inclusive, os hábitos alimentares. Algumas ocorrem por orientações médicas, outras ocorrem espontaneamente como forma de adaptação. Há um aumento pela preferência de alimentos moles, úmidos, pastosos e líquidos, assim como um aumento na quantidade de sal e/ou açúcar, decorrentes da diminuição do paladar ${ }^{10}, 11$.

Os movimentos mastigatórios ficam mais lentos e incoordenados, aumentando o tempo de preparo e o controle do bolo alimentar, consequentemente, surge a dificuldade para engolir, facilitando a ocorrência de problemas digestivos e nutricionais nestes indivíduos ${ }^{12}$. Idosos saudáveis conseguem adaptar-se às modificações trazidas pelo envelhecimento, ficando apenas predispostos a fragilização. Entretanto, idosos já fragilizados por doenças crônico-degenerativas, como a DA, podem apresentar alteração no processo de deglutição, ficando mais propensos a desnutrição, desidratação e aspiração, sinais clássicos de disfagia 1ํ. Pode-se enumerar uma série de sinais e sintomas indicativos de anormalidades no idoso: xerostomia (boca excessivamente seca) ou sialorréia (baba), dificuldade importante para mastigar e para manter o alimento na boca, movimentos repetitivos de língua e mandíbula durante a mastigação, dificuldade para iniciar a deglutição, mudança na postura de cabeça durante a deglutição, tosse e engasgos freqüentes. Também é comum a presença de refluxo nasal, asfixia freqüente e fadiga durante a alimentação, restos de alimentos não percebidos após a deglutição, pigarro constante, halitose, febres freqüentes e voz molhada durante a alimentação9 .

Atualmente há o reconhecimento de que a adoção de medidas de otimização, prevenção, reabilitação e manutenção são essenciais para a conquista de um envelhecimento saudável e melhoria da qualidade de vida do indivíduo com DA. Neste contexto, o conhecimento do estado de saúde em relação aos aspectos alimentares do idoso é importante para as políticas de saúde, pois auxilia os gestores na elaboração de estratégias específicas para essa população ${ }^{13}$. No Brasil, ainda há escassez de dados sobre as características de mastigação e deglutição nos indivíduos com a DA. Logo, as informações a respeito dessas questões poderão auxiliar no desenho de estratégias de promoção da saúde para a elaboração de programas de intervenção mais eficientes, melhorando a qualidade de vida da pessoa idosa e beneficiando o sistema de saúde. 0 objetivo desta pesquisa foi comparar as características das funções de mastigação e deglutição em idosos com e sem a Doença de Alzheimer.

\section{MÉTODO}

Foi realizado um estudo comparativo descritivo sobre as características das funções de mastigação e deglutição em idosos com e sem a Doença de Alzheimer. 
Os idosos foram alocados em dois grupos, controle e experimental. Para o grupo controle foram selecionados idosos saudáveis, com idade a partir de 60 anos, de ambos os sexos, participantes do "Programa Terceira Idade em Ação" (PTIA) da Universidade Federal do Piauí (UFPI). A seleção do grupo experimental se deu da seguinte forma: idosos com diagnóstico clínico de Doença de Alzheimer, segundo os critérios do Ministério da Saúde, regularmente cadastrados no "Programa de Medicamentos de Dispensação em Caráter Excepcional" do Ministério da Saúde, na cidade de Teresina-PI.

O cálculo da amostra foi realizado tomando-se por base a população de idosos do Piauí (290.410 idosos) ${ }^{14}$ e do número de idosos com DA no estado, cadastrados no "Programa de Medicamentos de Dispensação em Caráter Excepcional" (1.123 idosos com DA em outubro de 2008). Desta forma, chegou-se ao valor de $n(n=33)$. Foi adotado intervalo de confiança de $95 \%$ e margem de erro de $2 \%$. Ao valor inicial de $\mathrm{n}$ foram acrescidos 10 idosos em cada grupo, perfazendo um total de 43 idosos para cada grupo.

O estudo foi executado no período de julho de 2009 a fevereiro de 2010. Inicialmente, por meio de levantamento de endereço e telefone, realizou-se o contato com a família e/ou responsável do paciente para o esclarecimento da pesquisa e agendamento da visita em domicílio para a execução da coleta de dados.

Participaram do estudo indivíduos com idade igual ou superior a 60 anos, independente de raça ou sexo. O limite etário foi estabelecido em consonância com a Organização Mundial da Saúde ${ }^{15}$ que, nos países em desenvolvimento, classifica como idoso o adulto com 60 anos ou mais. Foram excluídos do estudo os seguintes pacientes usuários de via alternativa de alimentação (sonda enteral, gastrostomia), além de portadores de outras doenças concomitantes que pudessem interferir nos resultados (doenças neurodegenerativas em geral e depressão).

A função cognitiva dos idosos foi avaliada com a aplicação da Escala de Avaliação Clínica da Demência (Clinical Dementia Rating - CDR), em ambos os grupos. A CDR é um dos principais métodos para quantificar os diversos graus de demência e seu estadiamento. O questionário contém 10 itens, sendo seis para o informante, referentes ao idoso em relação à memória, orientação, capacidade de julgamento e solução de problemas, atividades domésticas e de lazer e cuidados pessoais; e quatro questões para o idoso: memória, orientação, juízo crítico e solução de problemas. Cada uma dessas seis categorias deve ser classificada em: 0 (nenhuma alteração); 0,5 (demência questionável); 1 (demência leve); 2 (demência moderada) e 3 (demência grave), exceto a categoria cuidados pessoais, que não tem o nível 0,5 . A categoria memória é considerada principal, ou seja, com maior significado e as demais categorias são secundárias ${ }^{16}$.

A classificação final da CDR é obtida pela análise dessas classificações por categorias, seguindo um conjunto de regras validadas ${ }^{17}$. Para se chegar à classificação não há necessidade de notas de corte estabelecidas pelo desempenho populacional, pois os indivíduos são comparados ao seu próprio desempenho passado. Apresenta sensibilidade de $91,2 \%$ e especificidade de $100 \%$ e é usualmente utilizada para classificar a gravidade da demência, relacionando as perdas cognitivas com a habilidade nas atividades de vida diária ${ }^{18}$.

A escala de CDR vem sendo mais utilizada nos programas e pesquisas no Brasil, por conseguir integrar aspectos cognitivos e comportamentais e o quanto estes aspectos interferem nas atividades de vida diária ${ }^{18}$, sendo, inclusive, uma exigência do "Programa de Medicamentos de Dispensação em Caráter Excepcional" do Ministério da Saúde para a dispensação de medicamentos aos pacientes com Doença de Alzheimer ${ }^{19,20}$.

A caracterização das funções de mastigação e deglutição foi obtida a partir da aplicação de Protocolo adaptado da Avaliação Miofuncional Orofacial da UNIFESP - Universidade Federal de São Paulo - Escola Paulista de Medicina 21,22. Para a avaliação da mastigação e deglutição foram utilizados alimentos nas consistências sólida (biscoito tipo "água e sal") e líquida (água).

Além das questões fonoaudiológicas específicas, o protocolo consta ainda de dados pessoais dos pacientes, tais como idade, sexo, escolaridade, profissão, data do provável diagnóstico, quando positivo, e tratamentos realizados. Foi aplicado nos idosos, com auxílio dos cuidadores, nos casos em que os pacientes não possuíssem capacidade para responder as perguntas.

Inicialmente, foi realizada inspeção da dentição e/ou próteses dos pacientes. Utilizou-se luvas de procedimento para a observação da cavidade oral e, com o auxílio de espátula de madeira, verificouse a presença e o número total de dentes, uso de prótese dentária e se esta era superior e/ou inferior, total ou parcial.

Para a caracterização da mastigação, foram observados os seguintes aspectos: restrição de consistências alimentares, tipo de corte do alimento, oclusão labial, velocidade, padrão mastigatório e movimentos mandibulares predominantes. A mastigação fisiológica deve ocorrer da seguinte forma: 
corte do alimento com os incisivos, lábios ocluídos, sem ruídos, com lateralização do alimento e padrão bilateral e alternado e com movimentos rotatórios de mandíbula ${ }^{21}$. A literatura relata diversos estudos sobre a avaliação da velocidade de mastigação baseados no tempo de mastigação de alimentos de consistências variadas. Há relatos de correlação entre a duração da mastigação obtida na avaliação com o cronômetro e a duração da mastigação obtida na avaliação por meio de Eletromiografia $(E M G)^{23,24,25}$. Com o auxílio de um cronômetro, verificou-se o tempo de mastigação em segundos para a consistência sólida (biscoito tipo "água e sal"). Foi considerado o período compreendido entre o corte do alimento e o início da primeira deglutição, observada pela elevação da laringe, característica do início da fase faríngea da deglutição, como manobra para proteção das vias aéreas ${ }^{24}$. Do tempo total, foi descontado 1 segundo relativo à fase oral. Foi considerada normal a velocidade de mastigação com duração semelhante ao tempo médio de mastigação dos grupos pesquisados.

A deglutição foi caracterizada a partir dos seguintes parâmetros: reflexo de deglutição, presença de deglutições múltiplas, auxílio de líquidos durante a deglutição, presença e frequência de engasgos e tosse, presença de resíduos alimentares na cavidade oral e dificuldade em deglutir comprimidos. Também foram questionados quanto a: perda de peso, mudança de dieta, tempo médio gasto nas refeições e postura habitual durante a alimentação. A perda de peso dos idosos, apesar de importante indicativo do estado nutricional, foi apenas questionada, tendo em vista a dificuldade prática de pesagem no grupo de idosos portadores de DA.

A deglutição, que pode ser iniciada conscientemente, deve ter duração média de 3 a 8 segundos, destes apenas 1 segundo corresponde à fase oral. Após a preparação do bolo alimentar, este é levado para trás, pelo movimento ondulatório da língua, tocando os pilares anteriores, desencadeando o reflexo propriamente dito ${ }^{21}$. A fase oral tem início com a propulsão posterior da língua, cujo perímetro se mantém em contato com os rebordos alveolares. Em seguida, a ponta da língua se eleva até o contato com a papila incisiva, seu corpo desliza contra o palato duro e sua base se deprime. A cavidade oral é fechada anteriormente pela aproximação dos lábios e elevação da mandíbula. Nesse momento, tem-se a contração do músculo milohióideo, que permite uma elevação maciça da língua e, então, o bolo é direcionado para a orofaringe. Nos indivíduos normais, no momento em que a língua entra em contato com o palato duro até a elevação do hióide, tem-se o início da fase faríngea e estas ações passam-se em um segundo ${ }^{21,23}$. A avaliação da deglutição tem como padrão-ouro a Videofluoroscopia da Deglutição. Entretanto, tal procedimento ainda é uma realidade distante no país, especialmente no centro onde a pesquisa foi desenvolvida. Desta forma, a avaliação desta função foi realizada pela observação clínica. Considerou-se normal a deglutição cujo reflexo foi iniciado cerca de 1 segundo após a conclusão da mastigação, tendo como marco a elevação da laringe.

O Comitê de Ética em Pesquisa da Universidade Federal do Piauí aprovou o projeto em seus aspectos éticos e metodológicos de acordo com as Diretrizes estabelecidas na Resolução 196/96 e complementares do Conselho Nacional de Saúde, CAAE (Certificado de Apresentação para Apreciação Ética): 0197.0.045.000-08.

Os participantes ou responsáveis assinaram um Termo de Consentimento Livre Esclarecido e descritivo da pesquisa ao qual foram submetidos (Apêndice A), elaborado de acordo com a "Declaração de Helsinque III", capítulo 50, parágrafos $50.20 / 27$.

Os dados foram organizados em planilhas do Programa Microsoft Excel, versão 10.0 XP, para a criação de um banco de dados e realização de uma análise descritiva das variáveis observadas nos grupos estudados. Posteriormente, tais dados foram exportados para o programa SPSS (versão 10.0 for Windows) para análise estatística dos resultados. Foram utilizadas medidas de tendência central e de dispersão, como média e desvio padrão.

As associações entre as variáveis foram testadas usando os testes $t$ para amostras independentes e Qui-quadrado. O teste $t$ foi utilizado para verificar se havia diferença estatística entre as variáveis quantitativas. $\mathrm{Na}$ análise das variáveis possivelmente inter-relacionadas, foi utilizado o coeficiente de correlação de Pearson. O teste Qui-quadrado foi empregado para verificar se havia associação entre as variáveis qualitativas. A diferença foi considerada significante quando $p<0,05$ e o intervalo de confiança adotado foi de $95 \%$. 


\begin{tabular}{|c|c|c|c|c|c|}
\hline & $\begin{array}{l}\text { Saudável } \\
\text { CDR } 0\end{array}$ & $\begin{array}{l}\text { Demência } \\
\text { questionável } \\
\text { CDR } 0,5\end{array}$ & $\begin{array}{c}\text { Demência leve } \\
\text { CDR } 1\end{array}$ & $\begin{array}{l}\text { Demência } \\
\text { moderada } \\
\text { CDR } 2\end{array}$ & $\begin{array}{l}\text { Demência } \\
\text { grave } \\
\text { CDR } 3\end{array}$ \\
\hline MEMÓRIA & $\begin{array}{l}\text { Sem perda de } \\
\text { memória, ou } \\
\text { apenas } \\
\text { esquecimento } \\
\text { discreto e } \\
\text { inconsistente }\end{array}$ & $\begin{array}{l}\text { Esquecimento leve } \\
\text { e consistente; } \\
\text { lembrança parcial } \\
\text { de eventos; } \\
\text { "esquecimento } \\
\text { benigno" }\end{array}$ & $\begin{array}{c}\text { Perda de memória } \\
\text { moderada, mais } \\
\text { acentuada para } \\
\text { fatos recentes; o } \\
\text { déficit interfere } \\
\text { com atividades do } \\
\text { dia a dia }\end{array}$ & $\begin{array}{l}\text { Perda de memória } \\
\text { grave; apenas } \\
\text { material muito } \\
\text { aprendido é retido; } \\
\text { materiais novos } \\
\text { são rapidamente } \\
\text { perdidos }\end{array}$ & $\begin{array}{l}\text { Perda de memória } \\
\text { grave; apenas } \\
\text { fragmentos } \\
\text { permanecem }\end{array}$ \\
\hline ORIENTAÇÃO & $\begin{array}{l}\text { Plenamente } \\
\text { orientado }\end{array}$ & $\begin{array}{l}\text { Plenamente } \\
\text { orientado }\end{array}$ & $\begin{array}{l}\text { Dificuldade } \\
\text { moderada com as } \\
\text { relações de tempo; } \\
\text { orientado no } \\
\text { espaço no exame, } \\
\text { mas pode ter } \\
\text { desorientação } \\
\text { geográfica em } \\
\text { outros locais }\end{array}$ & $\begin{array}{c}\text { Geralmente } \\
\text { desorientado }\end{array}$ & $\begin{array}{c}\text { Orientação pessoal } \\
\text { apenas }\end{array}$ \\
\hline $\begin{array}{l}\text { JULGAMENTO } \\
\text { E SOLUÇÃO DE } \\
\text { PROBLEMAS }\end{array}$ & $\begin{array}{c}\text { Resolve bem } \\
\text { problemas do dia a } \\
\text { dia, juízo crítico é } \\
\text { bom em relação ao } \\
\text { desempenho } \\
\text { passado }\end{array}$ & $\begin{array}{c}\text { Leve } \\
\text { comprometimento } \\
\text { na solução de } \\
\text { problemas, } \\
\text { semelhanças e } \\
\text { diferenças }\end{array}$ & $\begin{array}{c}\text { Dificuldade } \\
\text { moderada na } \\
\text { solução de } \\
\text { problemas, } \\
\text { semelhanças e } \\
\text { diferenças; } \\
\text { julgamento social } \\
\text { geralmente } \\
\text { mantido }\end{array}$ & $\begin{array}{c}\text { Gravemente } \\
\text { comprometido para } \\
\text { solução de } \\
\text { problemas, } \\
\text { semelhanças e } \\
\text { diferenças. Juízo } \\
\text { social geralmente } \\
\text { comprometido }\end{array}$ & $\begin{array}{l}\text { Incapaz de } \\
\text { resolver problemas } \\
\text { ou de ter qualquer } \\
\text { juízo crítico }\end{array}$ \\
\hline $\begin{array}{l}\text { ASSUNTOS NA } \\
\text { COMUNIDADE }\end{array}$ & $\begin{array}{c}\text { Função } \\
\text { independente na } \\
\text { função habitual de } \\
\text { trabalho, compras, } \\
\text { negócios, finanças, } \\
\text { e grupos sociais }\end{array}$ & $\begin{array}{l}\text { Leve dificuldade } \\
\text { nestas atividades }\end{array}$ & $\begin{array}{c}\text { Incapaz de } \\
\text { funcionar } \\
\text { independentement } \\
\text { e nestas atividades } \\
\text { embora ainda } \\
\text { possa } \\
\text { desempenhar } \\
\text { algumas; pode } \\
\text { parecer normal à } \\
\text { avaliação } \\
\text { superficial }\end{array}$ & $\begin{array}{c}\text { Sem possibilidade } \\
\text { de desempenho } \\
\text { fora de casa. } \\
\text { Parece } \\
\text { suficientemente } \\
\text { bem para ser } \\
\text { levado a atividades } \\
\text { fora de casa }\end{array}$ & $\begin{array}{l}\text { Sem possibilidade } \\
\text { de desempenho } \\
\text { fora de casa. } \\
\text { Parece muito } \\
\text { doente para ser } \\
\text { levado a atividades } \\
\text { fora de casa }\end{array}$ \\
\hline $\begin{array}{c}\text { LAR E } \\
\text { PASSATEMPOS }\end{array}$ & $\begin{array}{l}\text { Vida em casa, } \\
\text { passatempos, e } \\
\text { interesses } \\
\text { intelectuais } \\
\text { mantidos }\end{array}$ & $\begin{array}{l}\text { Vida em casa, } \\
\text { passatempos, e } \\
\text { interesses } \\
\text { intelectuais } \\
\text { levemente } \\
\text { afetados }\end{array}$ & $\begin{array}{c}\text { Comprometimento } \\
\text { leve, mas evidente } \\
\text { em casa; } \\
\text { abandono das } \\
\text { tarefas mais } \\
\text { difíceis; } \\
\text { passatempos e } \\
\text { interesses mais } \\
\text { complicados são } \\
\text { também } \\
\text { abandonados }\end{array}$ & $\begin{array}{l}\text { Realiza as tarefas } \\
\text { mais simples. } \\
\text { Interesses muito } \\
\text { limitados e pouco } \\
\text { mantidos }\end{array}$ & $\begin{array}{l}\text { Sem qualquer } \\
\text { atividade } \\
\text { significativa em } \\
\text { casa }\end{array}$ \\
\hline $\begin{array}{l}\text { CUIDADOS } \\
\text { PESSOAIS }\end{array}$ & Plenamente capaz & Plenamente capaz & $\begin{array}{c}\text { Necessita } \\
\text { assistência } \\
\text { ocasional }\end{array}$ & $\begin{array}{l}\text { Requer assistência } \\
\text { no vestir e na } \\
\text { higiene }\end{array}$ & $\begin{array}{c}\text { Requer muito } \\
\text { auxílio nos } \\
\text { cuidados pessoais. } \\
\text { Geralmente } \\
\text { incontinente }\end{array}$ \\
\hline
\end{tabular}

Fonte: Adaptado de MORRIS, 1993, p. 2413.

Figura 1 - Avaliação Clínica de Demência (Clincal Dementia Ratinga - CDR) 


\section{PROTOCOLO DE AVALIAÇÃO MIOFUNCIONAL OROFACIAL}

DATA:

\section{DADOS DE IDENTIFICAÇÃO}

Nome:

Data de Nascimento: Idade:

Endereço:

Telefone:

Celular:

Escolaridade:

Profissão:

Cuidador/responsável:

Data do diagnóstico:

II. TRATAMENTOS/ACOMPANHAMENTOS REALIZADOS

( ) Fono ( ) Neuro ( ) Nutrição ( ) Fisio ( ) Psicologia ( ) Psiquiatria

( ) Outro(s)

III. ESTRUTURAS DE MASTIGAÇÃO E DEGLUTIÇÃO

1. Dentição

( ) Presente ( ) Ausente

Prótese Dentária: ( ) Sim （）Não
( ) Total Superior
( ) Total Inferior
( ) Parcial Superior
( ) Parcial Inferior

\section{Lábios}

Postura: ( ) Ocluídos ( ) Abertos ( ) Entreabertos ( ) Dificuldade de Vedamento

Tônus: Superior ( ) Normal

( ) Aumentado

( ) Reduzido

Inferior ( ) Normal

( ) Aumentado

( ) Reduzido

3. Língua

Aspecto: ( ) Normal

Postura: ( ) Superior

( ) Alargada

( ) Inferior

Tônus: ( ) Normal

( ) Aumentado

( ) Marcas Laterais

( ) Posição Interdental

( ) Reduzido

\section{Bochechas}

Aspecto: ( ) Simétricas

Postura: ( ) Volumosas

( )Assimétricas

( ) Marcas Internas

Tônus: ( ) Normal

( ) Caídas

( ) Aumentado

( ) Reduzido

IV. ASPECTOS GERAIS DE ALIMENTAÇÃO

1. Tempo médio para cada refeição
( ) Até 30 minutos
( ) Mais de 30 minutos

2. Mudança recente de dieta

( ) $\operatorname{Sim}($ ) Não

3. Perda de peso

( ) Sim ( ) Não

4. Postura durante a alimentação

( ) Sentada ( ) Inclinada

5. Auxílio de Líquido

( ) Sim ( ) Não 
6. Restrição de alguma consistência ( ) Sim （） Não

( ) Sólido ( ) Pastoso ( ) Líquido

7. Usa a mastigação

( ) Sim ( ) Não

V. AVALIAÇÃO FUNCIONAL DA MASTIGAÇÃO E DEGLUTIÇÃO

1. Mastigação

Alimento Utilizado:

Características:

1.1.1 Corte

( ) Corte do alimento manualmente

( ) Incisão do alimento com incisivos centrais

( ) Incisão do alimento com caninos e/ou pré-molares

1.1.2. Oclusão labial

( ) Sim ( ) Não

1.1.3Velocidade

( ) Normal ( ) Rápida ( ) Lenta

1.1.4 Movimentos mastigatórios:

( ) Unilateral ( ) Bilateral ( ) Bilateral alternado

1.1.5 Movimentos mandibulares

( ) Movimentos Verticais de Mandíbula

( ) Movimentos Rotatórios de Mandíbula

2. Deglutição

Alimentos Utilizados:

Características

2.1.1 Reflexo de deglutição

( ) Normal ( ) Alterado

( ) Lentificado ( ) Reflexo ausente

2.1.2 Deglutições múltiplas para limpeza total

( ) Sim ( ) Não

2.1.3 Engasgos

( ) Sim ( ) Não

Consistência(s)

( ) Freqüentes ( ) Esporádicos

2.1.4 Tosse

( ) $\operatorname{Sim}$ ( ) Não

Consistência(s)
( ) Freqüente
( ) Esporádica

2.1.5 Resíduos alimentares na cavidade oral após a deglutição

( ) Sim ( ) Não

2.1.6 Dificuldade para Deglutir Comprimidos

( ) Sim ( ) Não

Figura 2 - Protocolo de avaliação miofuncional orofacial

\section{RESULTADOS}

Foram estudados 86 idosos, de ambos os sexos, avaliados no domicílio, divididos em dois grupos, sendo 43 do grupo controle (sem a Doença de Alzheimer) e 43 do grupo experimental (com a
Doença de Alzheimer). Na tabela 1 está representada a distribuição dos idosos, segundo as suas características dentro de cada grupo.

Ao avaliar as condições de mastigação e deglutição nos grupos pesquisados, é importante observar alguns fatores que poderiam influenciar a 
Tabela 1 - Caracterização dos idosos do estudo, segundo os grupos pesquisados. Teresina, PI, Brasil, 2009

\begin{tabular}{lccccccc}
\hline \multirow{2}{*}{ Variáveis } & & \multicolumn{2}{c}{ Controle } & \multicolumn{2}{c}{ Experimental } & \multicolumn{2}{c}{ Total } \\
\cline { 3 - 7 } Idade (anos) & $\mathbf{N}$ & $\%$ & $\mathbf{N}$ & $\%$ & $\mathbf{N}$ & $\%$ \\
\hline \multirow{2}{*}{ Sexo } & $60-69$ & 22 & 51,1 & 03 & 6,9 & 25 & 29 \\
& $70-79$ & 14 & 32,5 & 17 & 39,5 & 31 & 36 \\
& 80 e mais & 07 & 16,2 & 23 & 53,4 & 30 & 34,9 \\
\hline \multirow{3}{*}{ Escolaridade } & Feminino & 33 & 76,7 & 28 & 65,1 & 61 & 70,9 \\
& Masculino & 10 & 23,2 & 15 & 34,9 & 25 & 29 \\
\hline \multirow{4}{*}{ Condfabeto } & 03 & 07 & 15 & 35 & 18 & 20,9 \\
& Fundamental & 17 & 39,5 & 18 & 41,8 & 35 & 40,7 \\
& Médio & 16 & 37,2 & 07 & 16,2 & 23 & 26,7 \\
& Superior & 07 & 16,2 & 03 & 07 & 10 & 11,6 \\
\hline Total & 0 & 39 & 90,7 & 00 & 00 & 39 & 45,3 \\
& 1 & 00 & 00 & 14 & 32,5 & 14 & 16,2 \\
& 2 & 00 & 00 & 10 & 23,2 & 10 & 11,6 \\
& 3 & 00 & 00 & 11 & 25,5 & 11 & 12,8 \\
& Outros & 04 & 9,3 & 08 & 18,6 & 12 & 14 \\
\hline
\end{tabular}

alimentação de uma forma geral, interferindo direta ou indiretamente na execução de tais funções, como aspectos gerais de alimentação, dentição e/ ou uso de prótese dentária e tônus muscular dos idosos.
No que diz respeito aos aspectos gerais de alimentação, foram observados: tempo médio de refeição, mudança de dieta, perda de peso, postura, auxílio de líquido, restrição de consistência e uso da mastigação, conforme a Tabela 2.

Tabela 2 - Aspectos gerais de alimentação nos grupos controle e experimental. Teresina, PI, Brasil, 2009

\begin{tabular}{lccccccc}
\hline \multirow{2}{*}{ Variáveis } & \multicolumn{2}{c}{ Controle } & \multicolumn{2}{c}{ Experimental } & \multicolumn{2}{c}{ Total } \\
\cline { 3 - 8 } & & $\mathbf{N}$ & $\%$ & $\mathbf{N}$ & $\%$ & $\mathbf{N}$ & $\%$ \\
\hline Tempo médio de & até 30 min. & 42 & 97,6 & 28 & 65,1 & 70 & 81,4 \\
refeição & mais de 30 min. & 01 & 2,4 & 15 & 34,8 & 16 & 18,6 \\
\hline Mudança de dieta & sim & 03 & 6,9 & 12 & 27,9 & 15 & 17,4 \\
& não & 40 & 93,1 & 31 & 72,1 & 71 & 82,6 \\
\hline Perda de peso & sim & 04 & 9,3 & 23 & 53,4 & 27 & 31,4 \\
& não & 39 & 90,7 & 20 & 46,6 & 59 & 68,6 \\
\hline Postura & sentada & 43 & 100 & 38 & 88,4 & 81 & 94,2 \\
& inclinada & 00 & 00 & 05 & 11,6 & 05 & 5,8 \\
\hline Auxílio de líquido & sim & 29 & 67,5 & 32 & 74,4 & 61 & 71 \\
& não & 14 & 32,5 & 11 & 25,6 & 25 & 29 \\
\hline Restrição de & sim & 00 & 00 & 13 & 30,2 & 13 & 15,2 \\
consistência & não & 43 & 100 & 30 & 69,8 & 73 & 84,8 \\
\hline Uso da mastigação & sim & 43 & 100 & 36 & 83,7 & 79 & 91,9 \\
& não & 00 & 00 & 07 & 16,3 & 07 & 8,1 \\
\hline Total & & 43 & 50 & 43 & 50 & 86 & 100 \\
\hline
\end{tabular}


A análise da dentição dos idosos, quanto ao número de dentes, evidenciou uma média de 8,2 \pm 10,4 dentes. Ao se observar a quantidade de dentes por grupo, encontra-se os seguintes valores médios:
$10,5 \pm 11,6$ dentes para o grupo controle e 5,9 \pm 8,7 dentes para o grupo experimental. A Tabela 3 mostra a caracterização dos grupos segundo a presença de dentes e/ou prótese dentária.

Tabela 3 - Caracterização dos grupos segundo a presença de dentes e/ou prótese dentária. Teresina, PI, Brasil, 2009

\begin{tabular}{lccccccc}
\hline \multirow{2}{*}{ Dentição } & & \multicolumn{2}{c}{ Controle } & \multicolumn{2}{c}{ Experimental } & \multicolumn{2}{c}{ Total } \\
\cline { 3 - 7 } & & $\mathbf{N}$ & $\mathbf{\%}$ & $\mathbf{N}$ & $\%$ & $\mathbf{N}$ & $\%$ \\
\hline Dentes & ausente & 16 & 37,2 & 23 & 53,4 & 39 & 45,3 \\
& presente & 27 & 62,8 & 20 & 46,6 & 47 & 54,7 \\
\hline Prótese superior & total/parcial & 34 & 79,1 & 24 & 55,9 & 58 & 67,5 \\
& não usa & 09 & 20,1 & 19 & 44,2 & 28 & 32,5 \\
\hline Prótese inferior & total/parcial & 29 & 67,5 & 17 & 38,7 & 46 & 53,5 \\
& não usa & 14 & 32,5 & 26 & 60,4 & 40 & 46,5 \\
\hline Total & & 43 & 50 & 43 & 50 & 86 & 100 \\
\hline
\end{tabular}

A Tabela 4 mostra a distribuição percentual das características de lábios, língua e bochechas, quando avaliado o tônus muscular.

As características de mastigação nos grupos estudados estão apresentadas na Tabela 5. Do grupo total estudado $(n=86), 8,1 \%(n=7)$ dos idosos não realizavam mastigação, utilizando apenas alimentos nas consistências pastosa e líquida. Desta forma, para a mastigação, foram considerados apenas 36 idosos no grupo experimental. Foram avaliadas as seguintes características mastigatórias: corte do alimento, oclusão labial, velocidade de mastigação, movimentos mastigatórios e movimentos mandibulares.

A caracterização da deglutição nos dois grupos foi realizada de acordo com os seguintes critérios: reflexo de deglutição, deglutições múltiplas, engasgos, tosse, resíduos alimentares na cavidade oral após deglutir e dificuldade para deglutir comprimidos. Os valores encontrados estão dispostos na Tabela 6.

Tabela 4 - Características de lábios, língua e bochechas quanto ao tônus muscular por grupo pesquisado. Teresina, PI, Brasil, 2009

\begin{tabular}{lccccccc}
\hline \multirow{2}{*}{ Variáveis } & & \multicolumn{2}{c}{ Controle } & \multicolumn{2}{c}{ Experimental } & \multicolumn{2}{c}{ Total } \\
\cline { 3 - 7 } & & $\mathbf{N}$ & $\%$ & $\mathbf{N}$ & $\%$ & $\mathbf{N}$ & $\%$ \\
\hline \multirow{2}{*}{ Lábios } & normal & 29 & 67,4 & 15 & 34,9 & 44 & 51,1 \\
& alterado & 14 & 32,5 & 28 & 65,1 & 42 & 48,8 \\
\hline \multirow{2}{*}{ Língua } & normal & 31 & 72,1 & 16 & 37,2 & 47 & 54,6 \\
& alterado & 12 & 27,9 & 27 & 62,8 & 39 & 45,3 \\
\hline \multirow{2}{*}{ Bochechas } & normal & 24 & 55,8 & 09 & 20,9 & 33 & 38,4 \\
& alterado & 19 & 44,2 & 34 & 79,0 & 53 & 61,6 \\
\hline Total & & 43 & 50 & 43 & 50 & 86 & 100 \\
\hline
\end{tabular}


Tabela 5 - Características de mastigação nos grupos controle e experimental. Teresina, PI, BRASIL, 2009

\begin{tabular}{lccccccc}
\hline \multirow{2}{*}{ Variáveis } & \multirow{2}{*}{ Características } & \multicolumn{2}{c}{ Controle } & \multicolumn{2}{c}{ Experimental } & \multicolumn{2}{c}{ Total } \\
\cline { 3 - 7 } & & $\mathbf{N}$ & $\%$ & $\mathbf{N}$ & $\%$ & $\mathbf{N}$ & $\%$ \\
\hline \multirow{2}{*}{ Corte } & normal & 88,3 & 38 & 55,5 & 20 & 73,4 & 58 \\
& alterado & 11,6 & 05 & 44,4 & 16 & 26,6 & 21 \\
\hline Oclusão & sim & 83,7 & 36 & 72,2 & 26 & 78,5 & 62 \\
labial & não & 16,3 & 07 & 27,7 & 10 & 21,5 & 17 \\
\hline \multirow{2}{*}{ Velocidade } & normal & 69,7 & 30 & 22,2 & 08 & 48,1 & 38 \\
& alterada & 30,3 & 13 & 77,8 & 28 & 51,9 & 41 \\
\hline Movimento & normal & 37,2 & 16 & 22,2 & 08 & 30,3 & 24 \\
mastigatório & alterado & 62,8 & 27 & 77,8 & 28 & 69,7 & 55 \\
\hline Movimento & normal & 53,5 & 23 & 22,2 & 08 & 39,2 & 31 \\
mandibular & alterado & 46,5 & 20 & 77,8 & 28 & 60,8 & 48 \\
\hline Total & & 54,4 & 43 & 45,6 & 36 & 100 & 79 \\
\hline
\end{tabular}

Tabela 6 - Características de deglutição nos grupos pesquisados. Teresina, PI, Brasil, 2009

\begin{tabular}{lccccccc}
\hline \multirow{2}{*}{ Variáveis } & \multirow{2}{*}{ Características } & \multicolumn{2}{c}{ Controle } & \multicolumn{2}{c}{ Experimental } & \multicolumn{2}{c}{ Total } \\
\cline { 3 - 7 } & & $\mathbf{N}$ & $\%$ & $\mathbf{N}$ & $\%$ & $\mathbf{N}$ & $\%$ \\
\hline \multirow{2}{*}{ Deglutição } & normal & 88,4 & 38 & 53,5 & 23 & 70,9 & 61 \\
& alterada & 11,6 & 05 & 46,5 & 20 & 29,1 & 25 \\
\hline \multirow{2}{*}{ Deglutições } & $\operatorname{sim}$ & 20,9 & 09 & 44,2 & 19 & 32,5 & 28 \\
múltiplas & não & 79,1 & 34 & 55,8 & 24 & 67,5 & 58 \\
\hline \multirow{2}{*}{ Engasgos } & $\operatorname{sim}$ & 41,8 & 18 & 65,1 & 28 & 53,5 & 46 \\
& não & 58,1 & 25 & 34,9 & 15 & 46,5 & 40 \\
\hline \multirow{2}{*}{ Tosse } & $\operatorname{sim}$ & 30,2 & 13 & 60,5 & 26 & 45,3 & 39 \\
& não & 69,8 & 30 & 39,5 & 17 & 54,7 & 47 \\
\hline \multirow{2}{*}{ Resíduos } & sim & 20,9 & 09 & 55,8 & 24 & 38,3 & 33 \\
\hline Dificuldade & não & 79,1 & 34 & 44,2 & 19 & 61,7 & 53 \\
comprimidos & sim & 25,5 & 11 & 53,5 & 23 & 39,5 & 34 \\
Total & não & 74,5 & 32 & 46,5 & 20 & 60,5 & 52 \\
\hline
\end{tabular}

\section{DISCUSSÃo}

Estudos afirmam que as mudanças nos hábitos alimentares surgem gradativamente, como forma de adaptação às situações de perdas com que os idosos se deparam, tais como a diminuição da saúde orgânica e a integridade do sistema estomatognático ${ }^{26,27}$.

O presente estudo comparou as características das funções de mastigação e deglutição entre idosos com e sem a Doença de Alzheimer. A observação dos aspectos gerais de alimentação entre os grupos evidenciou diferença estatística significante $(p<0,05)$ para mudança de dieta, postura e uso da mastigação.

Não foi possível observar presença ou ausência de associação para tempo médio de refeição, perda de peso e restrição de consistências alimentares entre os grupos, quando aplicado o teste de quiquadrado. A distribuição de quadrado só pode ser adequadamente usada para um tamanho mínimo de 5 indivíduos por casilas (células). Quando a mesma não é atingida, não é possível identificar presença ou ausência da associação, necessitando, portanto, estudos posteriores com tamanho superior de indivíduos. 
Não foi encontrada associação $(p>0,05)$ entre o hábito de ingerir líquidos entre os dois grupos. Cerca de $71 \%$ dos idosos pesquisados tem o hábito de ingerir líquido durante a alimentação, de tal modo que não há qualquer diferenciação estatística entre controle e experimento. Em estudo realizado sobre a relação entre perdas dentárias e queixas de mastigação, deglutição e fala em indivíduos adultos, a ingestão de líquido foi mencionada como um hábito adquirido, sem relação com outras alterações presentes ${ }^{28}$.

Não foi possível mostrar a relação direta entre CDR e tempo médio de refeição. Contudo, fica evidente que na CDR 0 , a grande maioria dos idosos situa-se no menor tempo $(97,4 \%)$, e, à medida que aumenta o valor de CDR, e, portanto, o grau de DA, diminui o número de idosos com tempo médio de refeição de até 30 minutos (CDR 1 e 2 com $68 \%$ e CDR 3 com 54,5\%).

A perda de peso também foi questionada entre os grupos pesquisados, sendo encontrada em mais da metade dos idosos do grupo experimental $(53,4 \%)$ e em menos de $10 \%$ do grupo controle. 0 hipocampo e a amígdala, afetados na DA, podem causar perda de peso e alterações no comportamento alimentar. A redução do peso também pode estar associada a: autonegligência, esquecimento de se alimentar, alterações no olfato e no paladar, perda de habilidades, aumento do gasto energético, além de descuido no trato com os doentes ${ }^{26}$.

Com o envelhecimento a prevalência de doenças crônicas aumenta, podendo colaborar para o surgimento de anorexia, aumento das necessidades nutricionais e consequente carência de vitaminas e micronutrientes ${ }^{27}$. A DA tem como achados clínicos freqüentes a perda de peso e caquexia, especialmente nos primeiros estágios, mesmo com dieta adequada ${ }^{7}$.

Diversos estudos, em vários países, têm demonstrado a alta prevalência de distúrbios nutricionais na população idosa. Estudo comparativo realizado em Taiwan, com pacientes portadores de DA e indivíduos sadios, avaliou fatores que poderiam interferir na mudança ponderal dos idosos. Mais da metade dos pacientes portadores de DA desenvolveram perda ponderal, além de serem significantemente mais magros que os sadios. Apresentaram estado nutricional inadequado, apesar de a ingestão energética não ter sido significantemente diferente entre os dois grupos. Ao final do estudo, associouse a perda ponderal em idosos com Doença de Alzheimer à diminuição do apetite ${ }^{29}$.

Foi encontrada ausência de mastigação em $16,3 \%$ dos idosos do grupo experimental com diferença estatística significante entre os grupos $(p<0,05)$. À medida que se envelhece, fica-se mais propenso à fadiga muscular por conta do envelhecimento e hipotonia das fibras dos músculos da mastigação ${ }^{30}$. Tal função também pode ser afetada pela ausência de dentes, problemas periodontais, atrofia dos músculos mastigatórios, uso de prótese dentária e diminuição da produção de saliva nos idosos ${ }^{31}$.

A quantidade reduzida de dentes foi observada nos dois grupos, sendo encontrada diferença estatística significante $(p<0,05)$ para a quantidade de dentes entre os grupos, com média de 10,5 $\pm 11,6$ dentes para o grupo controle e 5,9 $\pm 8,7$ dentes para o grupo experimental. Entretanto, não houve diferença quanto ao uso de prótese total/parcial, superior e/ou inferior.

O edentulismo observado na pesquisa é característica frequente na maioria dos idosos brasileiros, agravando-se ainda mais na presença de doenças associadas ${ }^{25}$. A perda parcial e/ou total dos dentes é inclusive, aceita pela sociedade em geral, como algo normal ou natural do envelhecimento. Entretanto, a perda de um dente apenas pode gerar um desequilíbrio nas relações oclusais entre os restantes, ocasionando efeitos negativos na realização das funções estomatognáticas, principalmente as observadas no presente estudo ${ }^{25}$. De acordo com estudo realizado em 2004, indivíduos com perda dentária tinham 2,7 vezes mais propensão à dificuldade de mastigação que as pessoas sem perdas, encontrando associação significante entre menor número de pares dentários na cavidade oral e maior probabilidade em apresentar dificuldade mastigatória ${ }^{32}$.

Há várias referências na literatura sobre a perda múltipla dos dentes e consequente substituição dos alimentos duros por aqueles de menor consistência, ou seja, aqueles mais fáceis de engolir sem mastigar, o que pode comprometer, a longo prazo, o estado nutricional do indivíduo devido ao baixo valor nutricional desses alimentos. Além disso, a perda dentária contribui para o padrão unilateral de mastigação, uma vez que os indivíduos tendem a mastigar do lado de maior número de dentes ${ }^{33-34}$.

Em estudo realizado com indivíduos de 42 a 67 anos, usuários de prótese dentária total e/ou parcial foram encontradas várias alterações mastigatórias, como no corte do alimento e indefinição de tipo mastigatório ${ }^{35}$.

Vários trabalhos têm sido relacionados à qualidade de vida e de saúde geral entre os idosos com a possibilidade de consumo de bons nutrientes que, em geral, exigem a presença de dentes naturais sadios ou de próteses dentárias bem adaptadas. Estas, quando não possuem boas condições de funcionamento e trituração dos alimentos, causam modificações nos hábitos alimentares, podendo levar ao enfraquecimento do organismo, aumento 
dos problemas digestivos por uma ineficiência na formação do bolo alimentar. A opção por dietas mais pastosas/macias, para superar problemas orais, em médio prazo, pode agravar o estado nutricional de idosos ${ }^{32,33}$.

A redução do tônus muscular pode relacionarse à diminuição da massa muscular e a mudança morfológica que diminui as unidades motoras ativas com o avanço da idade ${ }^{23}$.

O tônus muscular pode ser caracterizado como normal, aumentado ou reduzido ${ }^{21}$. Foi verificada alteração de tônus muscular nos dois grupos em todas as estruturas pesquisadas (lábios, língua e bochechas), com predomínio de tônus reduzido, porém, não foi possível identificar presença ou ausência de associação neste caso. Entretanto, ficou evidente, nos idosos pesquisados, a diferença percentual do tônus muscular entre os grupos, com redução do tônus muscular maior no grupo experimental e, neste grupo, fica clara tal diminuição conforme o grau de CDR do idoso avança.

Estudos apontam a maior propensão à fadiga muscular por conta do envelhecimento e hipotonia das fibras dos músculos da mastigação. A composição muscular sofre modificações, com decréscimo do número de unidades motoras, caracterizando-as com menos fibras de rápida contração restantes, resultando em diminuição do tônus das estruturas ${ }^{27,32}$

Quando avaliadas as características de mastigação e deglutição em idosos, é importante lembrar que sofrem modificações estruturais e funcionais. Em idosos saudáveis, tais alterações são vistas como adaptações do organismo às condições presentes, sem prejuízos significantes na execução das funções. Fala-se em mastigação e deglutição adaptadas, havendo, inclusive, o termo presbifagia para identificar a deglutição do idoso. Porém, na presença de doenças associadas, o quadro tende a se agravar e o organismo pode perder sua capacidade de adaptação, levando a complicações no processo alimentar ${ }^{10}$.

Não foi possível identificar presença ou ausência de associação para corte, oclusão labial e velocidade de mastigação. Entretanto, ao se observar os percentuais encontrados para estas variáveis, especialmente para corte e velocidade mastigatória, identifica-se diferenças consideráveis, com predomínio de características alteradas no grupo experimental (corte com caninos/pré-molares ou corte manual ao invés corte com incisivos, padrão considerado normal; velocidade rápida ou lentificada, com predomínio desta última).

No grupo pesquisado, considerando-se o valor da CDR, foi possível verificar diminuição progres- siva do uso do corte adequado (com incisivos) à medida que aumenta o valor da CDR.

Apenas $50,7 \%$ dos idosos apresentaram velocidade de mastigação dentro dos parâmetros de normalidade. No grupo estudado, foi possível identificar que este padrão, quando visto por grau de CDR, apresentou diminuição percentual importante à medida que a DA evolui. Em estudo realizado para avaliar a alimentação e deglutição de idosos com DA leve (CDR 1) e moderada (CDR 2), foi observada manipulação lenta do bolo alimentar, ou seja, velocidade alterada de mastigação, em 50\% dos indivíduos avaliados e necessidade de auxílio para cortar o alimento e leva-lo à boca em alguns idosos ${ }^{39}$.

Em relação aos movimentos mastigatórios, não houve diferença estatística significante $(p>0,05)$ entre os grupos experimental e controle. Os movimentos mastigatórios foram considerados normais para padrão bilateral alternado e alterados para os padrões unilateral e bilateral simultâneo. O predomínio de alterações nos dois grupos $(62,8 \%$ no controle e $77,8 \%$ no experimental) pode ser explicado pelas características dentárias e musculares encontradas.

$\mathrm{Na}$ avaliação dos movimentos mandibulares, foi considerado normal o movimento rotatório e alterado o movimento vertical de mandíbula. Foi encontrada diferença estatística significante para movimentos mandibulares $(p<0,05)$ entre os grupos.

Apesar de não haver consenso na literatura sobre o padrão predominante de mastigação em idosos, sabe-se que há predominância dos padrões alterados de mastigação (unilateral e bilateral simultâneo). Em relação aos movimentos mandibulares, as pesquisas apontam para o predomínio do padrão alterado em idosos. Tais características são consequência do próprio envelhecimento e da redução do tônus muscular das estruturas envolvidas, dificultando a coordenação da lateralização do bolo alimentar e dos próprios movimentos mandibulares necessários aos padrões adequados (mastigação bilateral alternada com movimentos rotatórios de mandíbula) ${ }^{27,39}$.

Os processos fisiológicos associados ao enveIhecimento alteram vários parâmetros da deglutição, porém, não há evidências que as mudanças relacionadas à idade tenham efeito adverso em idosos saudáveis. Nos estágios mais avançados das doenças neurológicas, a disfagia é um sintoma comumente observado. Nas demências, especialmente no Alzheimer, são observadas alterações na deglutição decorrentes do déficit cognitivo significativo, que altera os hábitos de alimentação, tornando os pacientes dependentes, antes mesmo do aparecimento dos sintomas de disfagia ${ }^{39}$. 
A caracterização da deglutição nos dois grupos foi realizada de acordo com os seguintes aspectos: reflexo de deglutição, deglutições múltiplas, engasgos, tosse, presença de resíduos na cavidade oral após deglutir e dificuldade para deglutir comprimidos. Foram encontradas diferenças estatísticas significantes $(p<0,05)$ quando comparados entre os grupos com e sem DA as seguintes variáveis: reflexo de deglutição, deglutições múltiplas, presença de resíduos e dificuldade para ingerir comprimidos.

O reflexo de deglutição apresentou-se alterado em $11,6 \%(n=5)$ dos idosos do grupo controle. Entretanto, no grupo experimental, o percentual de deglutição alterada chegou a 46,5\% ( $n=20)$. Aliada ao reflexo de deglutição, foi encontrado o artifício de deglutições múltiplas em 20,9\% (n=9) do grupo controle e $44,2 \%(n=19)$ do experimental. A presença de engasgos esporádicos ou frequentes durante a alimentação foi observada em 41,8\% $(n=18)$ do controle; já no grupo experimental, a frequência de engasgos foi de $65,1 \% \quad(n=28)$. A ocorrência de tosse esporádica ou frequente apareceu na seguinte proporção: 30,2\% ( $n=13)$ e $60,5 \%(n=26)$ para os grupos controle e experimental, respectivamente.

Sobre a ocorrência de resíduos na cavidade oral após a deglutição, foi encontrada em 20,9\% (n=9) dos idosos do grupo controle ao passo que, no grupo experimental, a proporção chegou a $55,8 \%(n=24)$. A dificuldade na ingestão de cápsulas/comprimidos foi referida por $25,5 \%(n=11)$ dos idosos do grupo controle e por $53,5 \%(n=23)$ do grupo experimental.

Não foi possível verificar diferença estatística para engasgos e tosse entre os grupos pesquisados. Entretanto, ao se observar a distribuição por grau de CDR, o percentual de engasgos e tosse freqüentes $(5,1 \%$ a $72,7 \%$ e $5,1 \%$ a $63,7 \%$, respectivamente) eleva-se à medida que a doença evolui.

A tosse é um reflexo protetivo cuja presença indica a ocorrência de penetração e/ou aspiração laríngea. Entre as várias causas da aspiração, destacam-se as doenças neurológicas, principalmente em idosos. O envelhecimento e suas consequências, cirurgias de cabeça e pescoço, depressão, demência, uso de fármacos, particularmente em idosos, também favorecem a aspiração silenciosa ${ }^{42}$.

Diversos trabalhos relatam que as alterações da deglutição estão associadas com ao grau da demência ${ }^{1,10,39,43}$.

$\mathrm{Na}$ avaliação da deglutição realizada em estudo com idosos, os indivíduos conseguiam compensar algumas alterações encontradas nas fases oral e faríngea da deglutição, como deglutição lentificada, deglutindo repetidas vezes até a limpeza total ${ }^{39}$.
Vários estudos investigaram a dinâmica da deglutição com diversas consistências alimentares em portadores de DA. Foram encontradas as seguintes alterações: reflexo de deglutição lentificado, aumento do tempo total da deglutição para líquidos, penetração/aspiração para líquidos, ajuda de líquido para ingerir sólidos e engasgos/tosses com líquidos e sólidos ${ }^{39,40}$.

No Canadá, após revisão de prontuários de 47 idosos com demência foram verificadas alterações significantes na deglutição de metade dos pacientes: mastigação ausente, esquecimento da deglutição, dificuldade com líquidos e presença de tosse/engasgos ${ }^{41}$.

No presente estudo, encontrou-se valores bem distintos quando se compara o percentual de presença de resíduos na cavidade oral pós-deglutição entre os grupos, variando de $20,5 \%$ na CDR 0 a $72,7 \%$ na CDR 3 . Fato semelhante ocorre com a dificuldade de deglutir comprimidos, variando de $25,6 \%$ na CDR 0 a 90,9\% na CDR 3. Apesar de não ser possível identificar presença ou ausência de diferença dentro do grupo experimental, devido ao tamanho da amostra, os percentuais apresentados apontam para um declínio progressivo das características de deglutição paralelo à evolução da patologia.

Em estudo da alimentação e deglutição de idosos com doença de Alzheimer leve e moderada, queixas como engasgos/tosses com alimentos sólidos e líquidos e dificuldades para engolir comprimidos, foram relatadas principalmente pelos cuidadores dos idosos com demência moderada. Os autores sugeriram que as fases oral e faríngea da deglutição estão alteradas desde o início da Doença de Alzheimer ${ }^{42}$.

Apesar de não ser possível identificar presença ou ausência de diferença dentro do grupo experimental, devido ao tamanho da amostra, os percentuais apresentados apontam para um declínio progressivo das características de deglutição paralelo à evolução da DA.

Em pesquisa que avaliou 25 indivíduos com doença de Alzheimer de grau moderado e grave por meio da videofluoroscopia, as análises estatísticas evidenciaram que as alterações da deglutição estavam associadas com o grau da demência. Foi detectada tendência a aspiração proporcional ao grau de demência ${ }^{43}$.

Ao investigar as funções relacionadas a alimentação e deglutição de idosos entre 70 e 91 anos com demência, a maioria dos indivíduos pesquisados apresentou dificuldades relacionadas ao momento da alimentação, como: inabilidade no preparo do alimento, manuseio de utensílios, na higiene oral, além de dependência em relação ao cuidador ${ }^{39}$. 
A demência gera uma série de mudanças nos hábitos dos idosos em geral, inclusive os alimentares: perversão do apetite, mudança da preferência dos alimentos, redução ou aumento da ingestão oral, uso inadequado de utensílios e incapacidade para expressar de forma adequada os sinais de sede, fome e saciedade. Com a progressão da doença, o nível de dependência aumenta, chegando ao ponto de, em geral, necessitarem do auxílio de outros para realizar tarefas antes facilmente executadas, sendo comum o auxílio de sugestões verbais e assistência parcial durante atividades de vida diária, como a alimentação.

\section{CONCLUSÃO}

As características de mastigação e deglutição, de maneira geral, sofrem maior comprometimento nos idosos com Doença de Alzheimer, quando comparados a idosos saudáveis. Foram encontradas diferenças importantes entre os grupos, demonstrando degeneração concomitante ao avanço da doença.

Ainda são escassos os estudos sobre as dificuldades alimentares na Doença de Alzheimer, bem como suas conseqüências para a saúde nutricional e geral do idoso. Desta forma, é imprescindível a realização de mais estudos relacionados a este tema. É preciso aprofundar os conhecimentos acerca da realização das funções de mastigação e deglutição na Doença de Alzheimer, inclusive em cada estágio da doença, buscando relações entre estas e o estado nutricional dos indivíduos, com vistas à promoção da saúde e otimização dos programas de intervenção, garantindo, assim, melhor qualidade de vida para a pessoa idosa, seja ela saudável ou portadora da Doença de Alzheimer.

\begin{abstract}
Purpose: to compare the characteristics of chewing and swallowing in elderly with and without AD. Method: a comparative study carried out with 86 elderly people, aged between 60 and 93 years, of both genders, distributed in control (without $A D, n=43)$ and experimental $(A D, n=43)$ groups. Cognitive function and staging of AD were evaluated by applying the CDR (Clinical Dementia Rating). For characterizing chewing and swallowing we applied the miofunctional assessment protocol. Results: the characteristics of chewing and swallowing have varied considerably when comparing both groups. There was a significant difference as for mandible movements, swallowing reflex, swallowing multiple residues and difficulty to swallow tablets. Conclusions: the characteristics of chewing and swallowing, in general, suffer more in AD patients when compared to healthy elderly.
\end{abstract}

KEYWORDS: Aged; Alzheimer Disease; Mastication; Deglutition

\section{REFERÊNCIAS}

1. Suzuki HS. Fonoaudiologia em Gerontologia. In: Conhecimentos essenciais para atender bem o paciente idoso. São José dos Campos: Pulso, 2003.

2. Cordei RC. Fisioterapia em Gerontologia. In: Suzuki HS. (Org.). Conhecimentos essenciais para atender bem o paciente idoso. São José dos Campos: Pulso, 2003.

3. Luzardo AR, Gorini MIPC, Silva APS. Características de idosos com Doença de Alzheimer e seus Cuidadores: uma série de casos em um serviço de Neurogeriatria. Texto Contexto Enferm. Florianópolis. 2006;15(4): 587-94.

4. Coelho FGM, Santos-Galduroz RF, Gobbi S, Stella

F. Atividade física sistematizada e desempenho cognitivo em idosos com demência de Alzheimer: uma revisão sistemática. Rev Bras Psiquiatr. 2009; 31(2):163-70.

5. Mansur LL, Carthbery MT, Caramelli P, Nitrini R. Linguagem e Cognição na Doença de Alzheimer. Psicologia: Reflexão e Crítica. 2005; 18(3): 300-7.

6. Mac-Kay APMG. Distúrbios de linguagem: demência. In:RussoIP. IntervençãoFonoaudiológica na Terceira Idade. São Paulo: Revinter, 2004.

7. Frank AA, Soares EA, Gouveia VE. Práticas Alimentares na Doença de Alzheimer. In: Frank AA, Soares EA. Nutrição no Envelhecer. São Paulo: Atheneu, 2004.

8. Gorzoni ML, Pires SL. Aspectos clínicos da demência senil em instituições asilares. Rev. Psiq. Clín. 2006; 33 (1); 18-23. 
9. Marchesan IQ. Distúrbios da Motricidade Oral. In: Russo IP. Intervenção Fonoaudiológica na Terceira Idade. São Paulo: Revinter, 2004.

10. Sanches EP, Suzuki HS. Fonoaudiologia em Gerontologia. In: Suzuki HS. (Org.). Conhecimentos essenciais para atender bem o paciente idoso. São José dos Campos: Pulso, 2003.

11. Silva LBC, Antunes AE, Paula A, Botelho MI, Silva AA, Amaya-Farfán J. Nutrition and dysphagia: body mass index, food consistency and food intake. Rev Bras Nutr Clin. 2008;23(2):91-6.

12. Campos MTFS, Monteiro JBR, Ornelas APRC. Fatores que afetam o consumo alimentar e a nutrição do idoso. Rev. Nutr. 2000; 13(3): 157-65.

13. Alves LC, Leite IC, Machado CJ. Perfis de saúde dos idosos no Brasil: análise da Pesquisa Nacional por Amostra de Domicílios de 2003 utilizando o método Grade of Membership. Cad. Saúde Pública. 2008; 24(3):535-46.

14. IBGE. Instituto Brasileiro de Geografia e Estatística. Contagem da população 2007. Rio de Janeiro, IBGE: 2007. Disponível em: www.ibge.gov. br. Acesso em: 07 Jul. 2008.

15. OMS. Organização Mundial de Saúde. Elementos traço na nutrição e saúde humana. São Paulo: Roca, 1998.

16. Maia ALG, Godinho C, Ferreira ED, Almeida V, Schuh A, Kaye J. Aplicação da versão brasileira da Escala de avaliação clínica da demência (clinical dementia rating - cdr) Em amostras de pacientes com demência. Arq Neuropsiquiatr 2006;64(2-B):485-9.

17. Morris J. The Clinical Dementia Rating (CDR): current version and scoring rules. Neurology. 1993;43 (11):2412-4.

18. Oliveira KCV, Barros ALS, Souza GFM. MiniExame do Estado Mental (MEEM) e Clinical Dementia Rating (CDR) em idosos com Doença de Alzheimer. Rev Neurocienc. 2008;16(2):101-6.

19. BRASIL, Ministério da Saúde. Protocolo Clínico e Diretrizes Terapêuticas da Doença de Alzheimer. Portaria SAS/MS no 843, de 31 outubro de 2002. Acesso em: 07 Jul. 2008. Disponível em: www. saude.gov.br.

20. Maia ALG, Godinho C, Ferreira ED, Almeida V, Schuh A, Kaye J. Aplicação da versão brasileira da Escala de Avaliação Clínica da Demência (Clinical Dementia Rating- CDR) em amostras de pacientes com demência. Arq Neuropsiquiatria. 2006;64(2-B):485-9.

21. Junqueira P. Avaliação Miofuncional. In: Marchesan IQ. Fundamentos em Fonoaudiologia: Aspectos Clínicos da Motricidade Oral. Rio de Janeiro: Guanabara Koogan, 1998.

22. Costa EG, Silva MCC, Costa MLG, Barros ALS, Soares RF. Análise da deglutição em sujeitos portadores de doença de Alzheimer. Rev Bras Otorrinolaringol. 2008; 74(1): Suplemento.

23. Melo, TM, Arrais, RD, Genaro, KF. Duração da mastigação de alimentos com diferentes consistências. Rev. Soc. Bras. Fonoaudiol. 2006; 11(3):170-4.

24. Saconato M, Guedes ZCF. Estudo da mastigação e da deglutição em crianças e adolescentes com Sequência de Möbius Rev Soc Bras Fonoaudiol. 2009;14(2):165-71.

25. Whitaker ME, Trindade Júnior AS, Genaro KF. Proposta de protocolo de avaliação clínica da função mastigatória. 2009;11(Supl.3):311-23.

26. Lima RMF, Amaral AKFJ, Aroucha EBL, Vasconcelos TMJ, Silva HJ, Cunha DA. Adaptações na mastigação, deglutição e fonoarticulação em idosos de instituição de longa permanência. Rev. CEFAC. 2009; 11( supl.3): 405-22.

27. Kossioni $A E$, Dontas AS. The stomatognathic system in the elderly. Useful information for the medical practitioner. Clin Interv Aging. 2007; 2(4):591-7.

28. Jorge TM, Bassi AKZ, Yarid SD, Silva HM, Silva RPR, Caldana ML et al. Relação entre perdas dentárias e queixas de mastigação, deglutição e fala em indivíduos adultos. Rev. CEFAC. 2009;11(supl.3):391-7.

29. Costa ACR, Rodrigues MCSG, Lima RR. Deficiência da capacidade mastigatória e sua influência sobre memória e aprendizagem - revisão de literatura. Revista Paraense de Medicina. 2006; 20(3): 51-4.

30. Vannucchi H, Cunha DF, Bernardos MM, Unamuno MRDL. Avaliação dos níveis séricos das vitaminas $A, E, C$ e B2, de carotenóides e zinco, em idosos hospitalizados. Rev. Saúde Pública. 1994;28 (2):121-6.

31. Machado JS, Frank AA, Soares EA. Fatores dietéticos relacionados à doença de Alzheimer. Rev Bras Nutr Clin. 2006; 21(3):252-7.

32. Suzuki HS. O entardecer da deglutição: um estudo sobre modificações nos hábitos da rotina alimentar x fisiologia da deglutição do idoso normal. [monografia] São Paulo (SP): CEFAC Pós-Graduação em Saúde e Educação; 1997.

33. Jales MA, Cabral RR, Silva HJ, Cunha DA. Características do sistema estomatognático em idosos: diferenças entre instituição pública e privada. Rev CEFAC. 2005;7(2):178-87.

34. Brennan DS, Spencer AJ, Roberts-Thomson KF. Tooth loss, chewing ability and quality of life. Qualit Life Res. 2008; 17(2):227-35.

35. Nowjack-Raymer RE, Sheiham A. Numbers of natural teeth, diet, and nutritional status in US adults. J Dent Res. 2007; 86(12):1171-5. 
36. Chauncey HH, Muench ME, Kapur KK, Wayler $\mathrm{AH}$. The effect of the loss of teeth on diet and nutrition. Int Dent J. 1984; 34(2):98-104.

37. Mesas AE, Andrade SM, Cabrera MAS, Bueno VLRC. Salud oral y déficit nutricional en adultos mayores no institucionalizados en Londrina, Paraná, Brasil. Rev. Bras. Epidemiol. 2010; 13(3): 434-45.

38. Cavalcanti RVA, Bianchini EMG. Verificação e análise morfofuncional das características da mastigação em usuários de prótese dentária removível. Rev. CEFAC. 2008;10(4): 490-502.

39. Sanches EP, Bilton T, Suzuki H, Ramos LR. Estudo da alimentação e deglutição de idosos com doença de Alzheimer leve e moderada. Distúrbios da Comunicação. 2003;15(1): 9-37.
40. Machado J, Caram CLB, Frank AA, Soares EA, Laks J. Estado nutricional na Doença de Alzheimer. Rev Assoc Med Bras. 2009; 55(2): 188-91.

41. Freitas MIA, Ribeiro AF, Chiba T, Mansur LL. Investigação fonoaudiológica de idosos em programa de assistência domiciliar. Einstein. 2007; 5(1):6-9.

42. Bernardo WM, Barrichello APC, Cecilio LB. Quando suspeitar de aspiração silenciosa? Rev Assoc Med Bras. 2007;53 (4): 283-92.

43. Humbert IA, McLaren DG, Kosmatka K, Fitzgerald $\mathrm{M}$, Johnson S, Porcaro $\mathrm{E}$ et al. Early deficits in cortical control of swallowing in Alzheimer's disease. J Alzheimers Dis. 2010.19(4):1185-97.

Endereço para correspondência:

Thaíza Estrela Tavares

R. Valdemar Martins, 3333, ap. 101 -

Morada do Sol - Teresina - PI

CEP: 64055-280

E-mail: testrela@zipmail.com.br tetavares@novafapi.com.br 